\title{
Fuzzy Controller Based QoS Routing Algorithm with a Multiclass Scheme for MANET
}

\author{
B. Sun, C. Gui, Q. Zhang, H. Chen
}

\author{
Baolin Sun, Chao Gui, Qifei Zhang \\ School of Computing \\ Hubei University of Economics \\ Wuhan 430205, China \\ E-mail: blsun@163.com, guichao@126.com, cheffly@gmail.com \\ Hua Chen \\ College of Science \\ Wuhan University of Science and Engineering \\ Wuhan 430073, China \\ E-mail: Qiuchen_1022@163.com
}

\begin{abstract}
A mobile ad hoc network (MANET) consists of a set of mobile hosts that can communicate with each other without the assistance of base stations. Due to the dynamic nature of the network topology and restricted resources, quality of service $(\mathrm{QoS})$ and multicast routing in MANET are challenging tasks which attract the interests of many people. In this paper, we present a fuzzy controller based QoS routing algorithm with a multiclass scheme (FQRA) in mobile ad hoc networks. The performance of this scheduler is studied using NS2 (Network Simulator version 2) and evaluated in terms of quantitative measures such as packet delivery ratio, path success ratio and average end-to-end delay. Simulations show that the approach is efficient, promising and applicable in ad hoc networks.
\end{abstract}

Keywords: Mobile ad hoc networks, Fuzzy controllers, Routing algorithm, QoS

\section{Introduction}

Mobile ad hoc networks (MANET) consist of mobile nodes that autonomously establish connectivity via multihop wireless communications. There is no use of a static network infrastructure such as base station or any centralized administration in MANET. In ad hoc network, if two nodes are not within radio range, all message communication between them must pass through one or more intermediate nodes. All the nodes are free to move around randomly, thus changing the network topology dynamically [1-10]. These type of networks have many advantages, such as self reconfiguration and adaptability to highly variable mobile characteristics like the transmission conditions, propagation channel distribution characteristics and power level. They are useful in many situations such as military applications, conferences, lectures, emergency search, and rescue operations. However, such benefits come with new challenges which mainly resides in the unpredictability of the network topology due to mobility of nodes and the limited available bandwidth due to the wireless channel. These characteristics demand a new way of designing and operating these type of networks. For such networks, an effective routing protocol is critical for adapting to node mobility as well as possible channel error to provide a feasible path for data transmission [1-10].

AODV is an on-demand distance vector routing protocol [2]. The protocol is well known for the use in ad hoc networks. The use of multicasting with the network has many benefits. Multicasting reduces the communication cost for applications that sending the same data to many recipients [3, 5-8]. Instead of sending via multiple unicast, multicast reduces the channel bandwidth, sender and router processing and delivery delay. In addition, multicast gives robust communication whereby the receiver address is unknown or modifiable without the knowledge of the source within the wireless environment. 
Quality of service (QoS) support for multimedia applications is closely related to resource allocation, the objective of which is to decide how to reserve resources such that QoS requirements of all the applications can be satisfied [3, 6-11, 16, 21]. The goals of QoS routing are twofold: finding a suitable route through the network between the source and the destination that will have the necessary resources available to meet the QoS constraints; and achieving global efficiency in the utilization of resources. However, it is a significant technical challenge to provide reliable high-speed end-to-end communications in these networks, due to their dynamic topology, distributed management, and multi-hop connections. The provision of QoS requirements is of utmost importance for the development of future networks. For supporting QoS-aware applications, QoS based routing algorithms such as ticket base routing (TBR) [3] and core extraction dynamic source routing (CEDAR) [4] are proposed. In CEDAR, each host compares degree with its neighbors. The host with the largest degrees among its neighbors is selected as the core node. The core node is responsible for recording all information for its members. When a source host requests to establish a QoS routing path to some destination host, its core node must construct a QoS routing path. The advantage of core-based management is that it reduces the number of control packets during path construction. Chang proposes a two-level management approach for efficiently constructing and maintaining a QoS routing path in ad hoc wireless networks, significantly reducing the quantity of control packets [5]. In the first phase, the mobile hosts are partitioned into a number of complete graphs, each represented by a supernode managed by an agent. In the second phase, some agents of a larger degree than neighboring agents are selected as core nodes. Lorenz and Orda demonstrate in the literature [16] that this uncertainty places additional constraints on QoS provisioning. Xiao et al proposed a Dynamic Backup Routes Routing Protocol (DBR2P), a backup node mechanism for quick reconnection during link failures [20]. DBR2P is an on-demand routing protocol and it can set up many routes to reach a destination node in a given period. These algorithms determine a path that satisfies the required QoS.

Fuzzy logic exploits the pervasive imprecision, uncertainty and partial truth of the real world using simple linguistic statements and thereby achieves tractability, robustness, and low solution cost [11, 1319, 23]. Fuzzy logic based decision algorithm influences caching decisions of multiple paths uncovered during route discovery and avoids low quality paths [11, 14-19]. Differentiated resource allocation considering message type and network queue status is evaluated using fuzzy logic scheme [11]. Fernandez, $\mathrm{Hu}$, kazemian, Zhang et al propose the use of fuzzy logic controllers for the dynamic reconfiguration of edge and core routers [13-15, 23]. This reconfiguration allows for adjusting the network provisioning according to the incoming traffic and the QoS level achieved. Hu and Peter, utilize a self tuning fuzzy controller to apply an end-to-end rate-based feedback flow control algorithm for the available bit rate (ABR) service in ATM [14]. Sheng, et al propose an adaptive routing algorithm in which the link cost was dynamically assigned using a fuzzy system [18]. Their results show the traffic in the networks is rerouted to have less congestion or spare capacity. Sheu and Chen propose a fuzzy bandwidth allocation controller (FBAC) to support services including restricted time-bounded service such as voice and video in wireless networks [19]. Their results show the traffic in the network is rerouted to have less congestion or spare capacity. In those studies, the parameters and rules were further calibrated to obtain a more efficient evaluation. Because the ad hoc network traffics have self-similarity, a common statistical method has been widely used to verify self-similarity of time-series, so that the characteristics of the ad hoc wireless networks can be grasped.

In this paper, we present a Fuzzy controller based QoS Routing Algorithm with a multiclass scheme (FQRA) in mobile ad hoc networks. FQRA proposes to deal with route table management for keeping the active routes' lifetime. FQRA applies a fuzzy logic system to dynamically evaluate the route expiry time. The fuzzy logic is chosen because there are uncertainties associated with node mobility and the estimation of link crash; moreover, there is a mathematical model capable of estimating the node mobility. In addition, FQRA is able to take some controlling factors into consideration. Therefore, FQRA is a multiclass scheme fuzzy evaluation for QoS routing protocol. The performance of the FQRA is studied using NS2 [24] and evaluated in terms of quantitative measures such as improved path success ratio, 
reduced average end-to-end delay and increased packet delivery ratio.

The rest of the paper is organized as follows. In Section 2, we introduces the ad hoc network model and route issues. In Section 3, we presents the fuzzy QoS controller. In Section 4, we evaluate the performance of the algorithm and present the simulation results. Finally, Section 5 concludes the summary of the work and future challenges.

\section{Network Model and Routing Issues}

A network is usually represented as a weighted digraph $G=(N, E)$, where $N$ denotes the set of nodes and $E$ denotes the set of communication links connecting the nodes. $|N|$ and $|E|$ denote the number of nodes and links in the network respectively [3-11, 16]. In $G(N, E)$, considering a QoS constrained multicast routing problem from a source node to multi-destination nodes, namely given a non-empty set $M=\left\{s, u_{1}, u_{2}, \ldots, u_{m}\right\}, M \subseteq N, s$ is source node, $U=\left\{u_{1}, u_{2}, \ldots, u_{m}\right\}$ is a set of destination nodes. Multicast tree $T=\left(N_{T}, E_{T}\right)$, where $N_{T} \subseteq N, E_{T} \subseteq E$, if $C(T)$ is the cost of $T, P_{T}(s, u)$ is the path from source node $s$ to destination $u \in U$ in $T, D_{T}(s, u)$ and $B_{T}(s, u)$ are the delay and usable bandwidth of $P_{T}(s, u)$.

Definition 1. The cost of multicast tree $T$ is:

$$
C(T)=\sum_{e \in E_{T}} C(e), e \in E_{T} .
$$

Definition 2. The bandwidth, and delay route of multicast tree $T$ is the value of link bandwidth, and delay in the path from source node $s$ to each destination node $u \in U$. i.e.

$$
\begin{gathered}
B_{T}(s, u)=\min \left(B(e), e \in E_{T}\right) . \\
D_{T}(s, u)=\max \left(\sum_{e \in P_{T}(s, u)} D(e), u \in U\right) .
\end{gathered}
$$

Definition 3. Assume the minimum bandwidth constraint of multicast tree is $B$ and the maximum delay constraint is $D$, given a multicast demand $R$; then, the problem of bandwidth and delay constrained multicast routing is to find a multicast tree $T$, satisfying:

(1) Bandwidth constraint: $B_{T}(s, u) \geq B, u \in U$.

(2) Delay constraint: $D_{T}(s, u) \leq D, u \in U$.

Suppose $S(R)$ is the set, and $S(R)$ satisfies the conditions above; then, the multicast tree $T$ which we find is:

$$
C(T)=\min \left(C\left(T_{S}\right), T_{s} \in S(R)\right)
$$

\section{Fuzzy QoS Controller}

\subsection{Fuzzy Logic Controller}

The fuzzy logic was introduced by Zadeh as a generalization of the boolean logic [22]. The difference between these logics is that fuzzy set theory provides a form to represent uncertainties; that is, it accepts conditions partially true or partially false. Fuzzy logic is a good logic to treat random uncertainty, i.e., when the prediction of a sequence of events is not possible.

Fuzzy logic control system is rule-based system in which a set of so-called fuzzy rules represents a control decision mechanism to adjust the effects of certain causes that come from the system. The aim of the fuzzy control system is normally to substitute for or replace a skilled human operator with a fuzzy rule-based system. Specifically, based on the current state of a system, an inference engine equipped 
with a fuzzy rule base determines an on-line decision to adjust the system behavior in order to guarantee that it is optimal in some certain senses.

There are generally two kinds of fuzzy logic controllers. One is the feedback controller, which is not suitable for the high performance communication networks. Another one, which is used in this paper, is shown in Figure 1. The output of the fuzzy logic controller in Figure 1 is used to tune the controlled system's parameters based on the state of the system. This control mechanism is different from the conventional feedback control and considered as an adaptive control [11, 14, 19, 23].

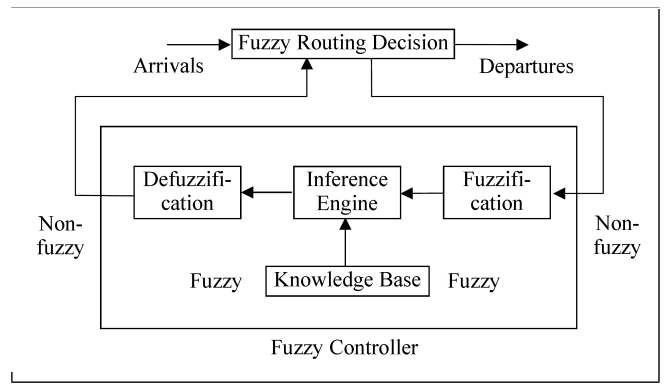

Figure 1: The fuzzy logic controller

The specific features of the fuzzy controller depend on the model under control and performance measurement. However, in principle, in the fuzzy controller we explore the implicit and explicit relationships within the system and subsequently develop the optimal fuzzy control rules as well as a knowledge base.

In the route discovery process, new routes are created. The creation of new routes makes use of forward packet (Table 1) and backward packet (Table 2). A forward packet is broadcasted by the sender and will be relayed by the neighbors of the sender.

When the forward packet reaches the destination node, it extracts the information collected by the forward packet and destroys it and subsequently creates a backward packet which follows the track of the forward packet, but in the reverse direction.

Table 1: Forward packet.

\begin{tabular}{lll}
\hline Source address & Destination address & Sequence number \\
\hline Number of hops & QoS Metric & Intermediate node \\
\hline
\end{tabular}

Table 2: Backward packet.

\begin{tabular}{lll}
\hline Source address & Destination address & Number of hops \\
\hline QoS Metric & Intermediate node & \\
\hline
\end{tabular}

The packets flow through router is presented in Figure 2. The aim was to decrease TCP and UDP traffic settling, rise and fall times; decrease overshoots and undershoots; and to stabilize throughput in the router's output connections to let the model adapt for load and link capacity variations. 


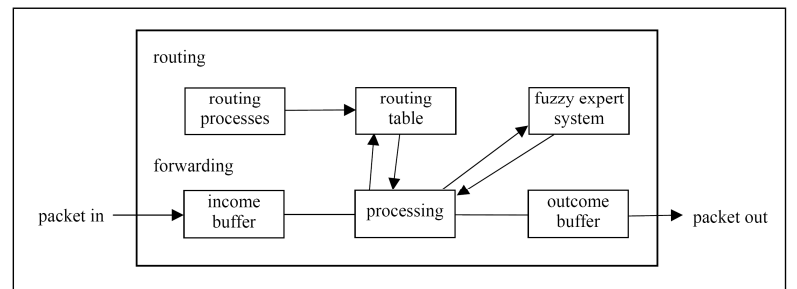

Figure 2: Schematic presentation of packets flow through router

\subsection{Scheduler Controller}

The packet scheduler used in our architecture is WRR (Weighted Round Robin). In this scheduler, queues are served according to a configurable weight that can be changed during network operation. This allows having control of the bandwidth assigned to each service class. The packet delay and discard rate for each queue (class) can be controlled by changing this weight. An example of membership function of schedule controller is showed in Figure 3. The fuzzy scheduler proposed here calculates the priority index of each packet. Here we consider all the inputs which decide the priority associated with the packet, unlike the previous scheduling schemes. Other membership functions are: packet delay in the expedited forwarding queue and discard rate due to queue overflow in the besteffort class. The output membership functions are also defined as trapezoid functions by the same previous reasons. We make use of the center of gravity defuzzification method, since it gives better results. The output membership function gives the weights assigned to each class in the WRR scheduler.

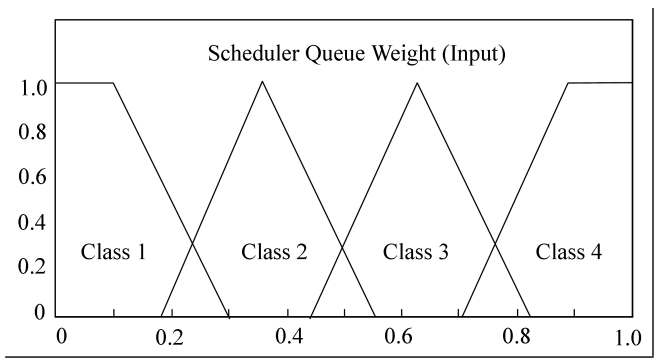

Figure 3: Scheduler membership functions

\subsection{Fuzzy Rule Base (Fuzzy Routing)}

Fuzzy systems reason with multi-valued fuzzy sets instead of crisp sets. The Fuzzy Logic Controller (FLC) (Figure 1) has two inputs: Residual Bandwidth and Traffic Class and one output: Fuzzy Routing Decision [12, 22].

Mamdani fuzzy-rule based systems constitute of a linguistic description in both the antecedent parts and the consequent parts. Each rule (Table 3) is a description of a condition-action statement that may be clearly interpreted by the users. Rule base is an IF-THEN rule group with fuzzy sets that represents the desired behavior of a fuzzy system. It can be defined in agreement with the administrative policy.

$$
\text { IF } x_{1} \text { is } A_{i 1} \text { and } \ldots \text { and } x_{n} \text { is } A_{i n} \text { THEN } y \text { is } C_{i}, i=1,2, \ldots, L
$$

where $L$ is the number of fuzzy rules, $x_{j} \in U_{j}, j=1,2, \ldots, n$, are the input variables, $y$ is the output variable, $A_{i j}$ are the fuzzy sets of the input linguistic variable $x_{j}$ and $C_{i}$ is called the set of the output linguistic variable $y . A_{i j}$ and $C_{i}$ are characterized by both membership functions.

Inputs are of the form: $x_{1}$ is $A_{1}^{\prime}, x_{2}$ is $A_{2}^{\prime}, \ldots, x_{n}$ is $A_{n}^{\prime}$ where $A_{1}^{\prime}, A_{2}^{\prime}, \ldots, A_{n}^{\prime}$ are fuzzy subsets of $U_{1}$, $U_{2}, \ldots U_{n}$, which are the universe of discourse of inputs. 
Table 3: Fuzzy rule base - QoS classes and application type.

\begin{tabular}{cl}
\hline QoS Class & Application Type \\
\hline 1 & Video conference \\
2 & SDTV-quality voice \\
3 & CD-quality audio \\
4 & High-quality voice \\
\hline
\end{tabular}

\section{Simulation}

\subsection{Random Graph Generation}

In generating random graphs, we have adopted the method used in Kazemian, where vertices are placed randomly in a rectangular coordinate grid by generating uniformly distributed values for their $x$ and $y$ coordinates [15]. The remaining edges of the graph are chosen by examining each possible edge $(u, v)$ and generating a random number $0 \leq r<1$. If $r$ is less than a probability function $P(u, v)$ based on the edge distance between $u$ and $v$, then the edge is included in the graph. The distance for each edge is the Euclidean distance denoted as $d(u, v)$ between the nodes that form the end-points of the edge. We use the probability

$$
P(u, v)=\beta \exp \left[-\frac{d(u, v)}{\alpha L}\right] .
$$

where $d(u, v)$ is geometric distance from node $u$ to node $v$ and $L$ is maximum distance between two nodes. The parameters $\alpha$ and $\beta$ are in the range $(0,1)$ and can be used to obtain certain desirable characteristics in the topology; parameter $\alpha$ can be used to control short edge and long edge of the random graph, and parameter $\beta$ can be used to control the value of average degree of the random graph.

\subsection{Simulation Model}

To conduct the simulation studies, we used randomly generated networks on which the algorithms were executed. This ensures that the simulation results are independent of the characteristics of any particular network topology. Using randomly generated network topologies also provides the necessary flexibility to tune various network parameters such as average degree, number of nodes, and number of edges, and to study the effect of these parameters on the performance of the algorithms. The platform used was the NS2 (Network Simulator version 2) [24]. NS2 is a discrete event simulator targeted at networking research. NS2 provides substantial support for simulation of TCP, routing, and multicast protocols over wired and wireless networks.

Fuzzy Routing Decision was implemented using the Fuzzy Logic Toolbox in MATLAB [25]. The simulator ran with various input configuration settings and the statistics collected were analyzed in comparison with other well-known on demand routing protocol AODV [2].

Our simulation modeled a network of mobile nodes placed randomly within $1000 \times 1000$ meter area. Each node had a radio propagation range of 250 meters and channel capacity of $10 \mathrm{Mbps}$. Two-ray propagation model was used. The IEEE 802.11 distributed coordination function was used as the medium access control protocol. A random waypoint mobility model was used: each node randomly selected a position and moves toward that location with a speed ranging from just above $0 \mathrm{~m} / \mathrm{s}$ to $10 \mathrm{~m} / \mathrm{s}$. When the node reached that position, it became stationary for a programmable pause time; then it selected another position and repeated the process. The simulation was repeated with different seed values. A traffic 
generator was developed to simulate CBR (Constant Bit Rate) sources. The size of the data payload was 512 bytes. Data sessions with randomly selected sources and destinations were simulated. Each source transmitted data packets at a minimum rate of 4 packets/sec. and maximum rate of 10 packets $/ \mathrm{sec}$. Traffic classes were randomly assigned and simulation was carried out with different bandwidth requirements. There were no network partitions throughout the simulation. Each simulation was executed for 600 seconds of simulation time. Multiple runs with different seed values were conducted for each scenario and collected data was averaged over those runs. Table 4 lists the simulation parameters which are used as default values unless otherwise specified.

Table 4: Simulation parameters

\begin{tabular}{ll}
\hline Number of nodes & 100 \\
Terrain range & $1000 \mathrm{~m} \times 1000 \mathrm{~m}$ \\
Transmission range & $250 \mathrm{~m}$ \\
Average node degree & $3-5$ \\
Node's mobility speed & $0-10 \mathrm{~m} / \mathrm{s}$ \\
Mobility model & Random way point \\
Propagation model & Free space \\
Channel bandwidth & $5 \mathrm{Mbps}$ \\
Links delay & $20-200 \mathrm{~ms}$ \\
Traffic type & CBR \\
Data payload & 512 bytes/packet \\
Node pause time & $0-10$ seconds \\
\hline
\end{tabular}

\subsection{Performance Measures}

The following measures are used in computing the scheduler performance. These measures were derived from one suggested by the MANET working group for routing protocol evaluation.

Packet delivery ratio: Packet delivery ratio is the ratio of the number of data packets actually delivered to the destinations to the number of data packets supposed to be received. This estimate gives us an idea about how successful the protocol is in delivering packets to the application layer. A high value of packet delivery ratio indicates that most of the packets are being delivered to the higher layers and is a good indicator of the algorithm performance.

Path success ratio: The ratio of the number of connection request discover to the destinations to the number of routed connection requests. The successful routing request is defined as the path computed and established by the algorithm satisfies the delay and bandwidth constraints. This number presents the effectiveness of the algorithm.

Average end-to-end delay: This indicates the end-to-end delay experienced by packets from source to destination. The average end-to-end packet delay is computed as the ratio of total end-to-end delays to the aggregate number of packets successfully delivered to the destination nodes during a simulation run. A higher value of end-to-end delay means that the network is congested and hence the routing algorithm does not perform well. 


\subsection{Simulation Results}

In this performance evaluation the following performance measures were evaluated: packet delivery ratio, percentile of path success ratio and edge-to-edge delay. For each evaluation, we used CBR. All simulations started with initial scheduler configuration with $60 \%$ of the bandwidth for each class. To eliminate simulation results with an empty network, we started collecting results 30 seconds after the beginning of the simulation.

After optimization procedure was executed, we could verify the result comparing packet delivery ratio, path success ratio, and average end-to-end delay.

Figure 4 shows the performance analysis of the packet delivery ratio vs. network size for the FQRA, AODV, and Non-QoS (not quality of service metric constraints) in ad hoc network. Using Non-QoS constraints algorithm as a base line for comparison, the result shows that both FQRA and AODV are much better than the Non-QoS constraints algorithm, FQRA is considerably better than AODV. This is because that AODV needs to rediscover the route to retransmit data packets that are lost due to the node's mobility or unreal route paths during the communication. The advantage of FQRA is resulted from choosing the right routing path or updating the unreal route paths just in time by the virtue of the suitable route lifetime estimation.

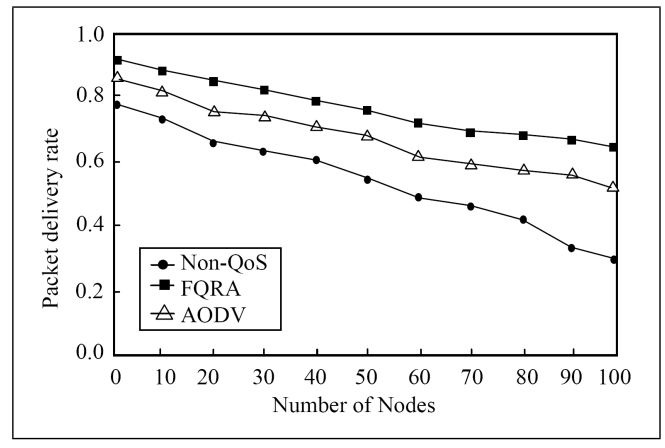

Figure 4: Packet delivery ratio vs. Networks size

Figure 5 shows the comparison of the three algorithms with respect to the average end-to-end delay vs. number of nodes. It shows that the end-to-end delay increases usually with the increasing node. From the Figure 5, we can see that when the nodes increase, FQRA algorithm average end-to-end delay is lower than that of AODV and Non-QoS algorithm. This is because the fuzzy scheduler controller has stability routes and gives more precedence to the packets. Both the AODV and Non-QoS algorithm need more time and more control overhead than the FQRA does to recover unreal paths (broken paths) and to discover new paths. As the nodes increase, more packets are received and thus end-to-end delay increases. The end-to-end delay is important as many real time applications require a small latency to deliver usable data within stipulated period of time.

Figure 6 depicts a comparison path success rate to find the path through FQRA, AODV and Non-QoS in ad hoc network. With the relaxation of bandwidth constraints, the path success rate becomes higher for Non-QoS. The success rate is still higher than that of Non-QoS, which means more suitable for the routing choosing under timely data transmission application and dynamic network structure.

The average and-to-end delay performance as shown in the Figure 7, proves that the end-to-end delay improves when scheduler is included. As the mobility varies from $0-10 \mathrm{~m} / \mathrm{s}$, the fuzzy controllers scheduler provides an end-to-end delay reduced by around 0.01 seconds to 0.05 seconds. It shows that the end-to-end delay increases usually with the increasing speed. It also shows that FQRA is much better than the other two. Both the Non-QoS and the AODV need more time and more control overhead than the FQRA does to recover unreal paths and to discover new paths. 


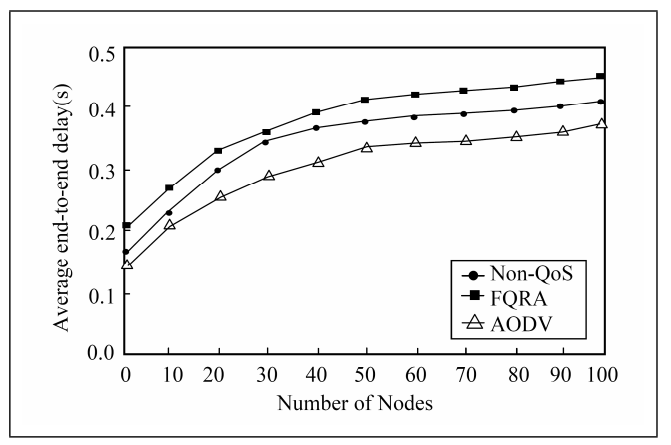

Figure 5: Average end-to-end delay vs. Networks size

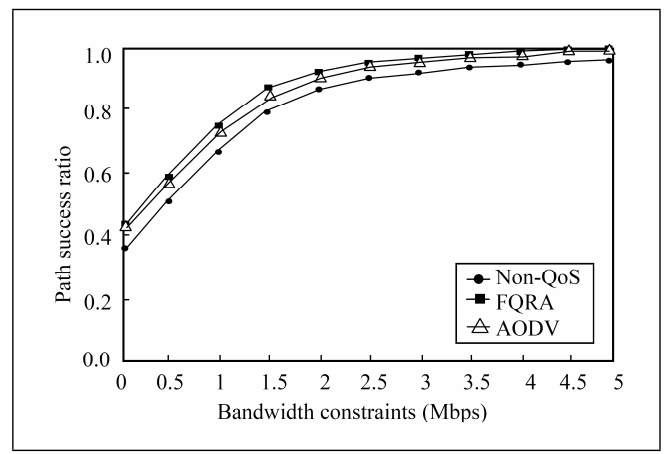

Figure 6: Path success ratio vs. Bandwidth

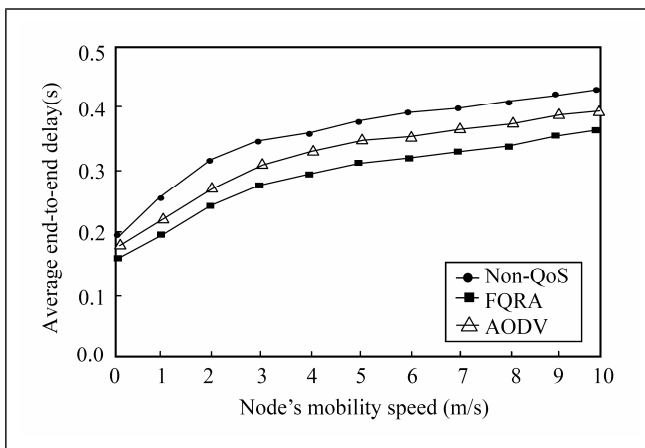

Figure 7: Average end-to-end delay vs. Node's mobility speed 


\section{Conclusion and Future Work}

Our QoS routing algorithm has produced significant improvements in throughput, average end-toend delay and path success ratio. The fuzzy controllers scheduler algorithm attaches a QoS class to each packet in the queue of the node. Unlike the normal sorting procedure for scheduling packet, the crisp QoS class is calculated by the fuzzy scheduler based on the above inputs which are derived from the network. The membership functions and rule bases of the fuzzy scheduler are carefully designed. The use of fuzzy logic improves the handling of inaccuracy and uncertainties of the ingress traffic into the domain.

In this paper, we present a fuzzy controller based QoS routing algorithm with a multiclass scheme in mobile ad hoc networks. The performance of this scheduler is studied using NS2 and evaluated in terms of quantitative measures such as path success ratio, average end-to-end delay and throughput. Simulation shows that the approach is efficient, promising and applicable in ad hoc networks.

Future work includes comparison with "crisp" versions of the fuzzy algorithm to isolate the contributions of fuzzy logic, as well as applications of fuzzy control to power consumption and directional antennas in MANETs. We also intend to compare FQRA with other QoS routing algorithm.

\section{Acknowledgement}

The authors thank the editors and the anonymous reviewers for their valuable comments that helped to improve the paper. This work is supported by China Postdoctoral Science Foundation of China (No. 20070410955), The Young and Middle-aged Elitists' Scientific and Technological Innovation Team Project of the Institutions of Higher Education in Hubei Province (No. T200902), and Key Scientific Research Project of Hubei Education Department (No. D20081904, Q20091903, B20091904).

\section{Bibliography}

[1] M. A. Rajan, M. G. Chandra, L. C. Reddy and P. Hiremath, Concepts of Graph Theory Relevant to Ad-hoc Networks. International Journal of Computers, Communications \& Control, Vol. 3, No. Suppl, pp. 465-469, 2008.

[2] C. Perkins, E. Belding-Royer and S. Das, Ad Hoc On-Demand Distance Vector (AODV) Routing. IETF RFC 3561, July 2003.

[3] S. Chen and K. Nahrstedt, Distributed Quality of Service routing in Ad hoc networks. IEEE Journal on Selected Areas in Communication, Vol. 17, No. 8, pp. 1488-1504, 1999.

[4] R. Sivakumar, P. Sinha and V. Bharghavan, CEDAR: Core Extraction Distributed Ad hoc Routing. IEEE Journal on Selected Areas in Communication, Vol. 17, No. 8, pp. 1454-1465, 1999.

[5] C. Y. Chang, C. T. Chang, T. S. Chen and H. R. Chang, Hierarchical management protocol for constructing a QoS communication path in wireless Ad Hoc networks. Information Sciences, Vol. 177, No. 13, pp. 2621-2641, 2007.

[6] B. L. Sun and L. Y. Li, A QoS Multicast Routing Optimization Algorithms Based on Genetic Algorithm, Journal of Communications and Networks, Vol. 8, No. 1, pp. 116-122, 2006.

[7] B. L. Sun, L. Y. Li, Q. Yang and Y. Xiang, An Entropy-Based Stability QoS Multicast Routing Protocol in Ad Hoc Network, Lecture Notes in Computer Science, Vol. 3947, Springer-Verlag, pp. 217-226, 2006. 
[8] B. L. Sun, S. C. Pi, C. Gui, et al, Multiple Constraints QoS Multicast Routing Optimization Algorithm in MANET based on GA. Progress in Natural Science, Vol. 18, No. 3, pp. 331-336, 2008.

[9] B. L. Sun, H. LI, Y. Zeng and Q. Q. QIN, Fuzzy QoS Controllers in Mobile Ad Hoc Networks using Genetic Algorithms. Journal of Computational Information Systems, Vol. 3, No. 6, pp. 2255-2260, 2007.

[10] B. L. Sun and L. Y. Li, A Distributed QoS Multicast Routing Protocol in Ad Hoc Networks. Journal of Systems Engineering and Electronics, Vol. 17, No. 3, pp. 692-698, 2006.

[11] A. Thomas, C. Chellappan and C. Jayakumar, ANTHOC - QoS: Quality of Service Routing in Mobile Ad Hoc Networks using Swarm Intelligence. in: The Second Asia Pacific Conference on Mobile Technology, Applications and Systems, Guangzhou, China, 15- 17 November, 2005.

[12] Y. H. Wang and C. F. Chao, Dynamic backup routes routing protocol for mobile ad hoc networks, Information Sciences, Vol. 176, No. 2, pp. 161-185, 2006.

[13] M. P. Fernandez, A. de Castro, P. Pedroza and J. F. de Rezende, QoS provisioning across a diffserv domain using policy-based management. in: Globecom 2001, San Antonio, USA, Nov. 2001.

[14] R. Q. Hu and D. Peter, A predictive self-tuning fuzzy logic feedback rate controller. IEEE/ACM Transactions on Networking, Vol. 8, No. 6, pp. 697-709, 2000.

[15] H. B. Kazemian and L. Meng, A fuzzy control scheme for video transmission in Bluetooth wireless. Information Sciences, Vol. 176, No. 9, pp. 1266-1289, 2006.

[16] D. H. Lorenz and A. Orda, Qos routing in networks with uncertain parameters. IEEE/ACM Transactions on Networking, Vol. 6, No. 6, pp. 768-778, 1998.

[17] S. Rea and D. Pesch, Multi-metric routing decisions for ad hoc networks using fuzzy logic. in: Proc. of 1st Intl. Symposium on Wireless Communication Systems, Mauritius, 20-22 September, pp. 403-407, 2004.

[18] H. M. Sheng, J. C. Wang, H. H. Huang and D. C. Yen, Fuzzy measure on vehicle routing problem of hospital materials. Expert Systems with Applications, Vol. 30, No. 2, pp. 367-377, 2006.

[19] S. T. Sheu and M. H. Chen, A fuzzy bandwidth allocation controller to support real-time traffic over wireless network. in: Proc. of IEEE Wireless Communications and Networking Conference, New Orleans, LA, Vol. 1, pp. 1348-1352, 1999.

[20] Y. Xiao, C. L. P. Chen and Y. Wang, Optimal admission control for multi-class of wireless adaptive multimedia services, IEICE Transaction on Communications, special issue on Mobile Multimedia Communications, Vol. E84-B, No. 4, pp. 795-804, 2001.

[21] B. L. Ye, M. Y. Guo, D. X Chen and S. L. Lu, A degree-constrained QoS-aware routing algorithm for application layer multicast, Information Sciences, Vol. 177, No. 17, pp. 3613-3626, 2007.

[22] L. A. Zadeh, Fuzzy sets. Information and Control, Vol. 8, No. 3, pp. 338-353, 1965.

[23] R. Zhang and Y. Phillis, Fuzzy Control of Queueing System with Heterogeneius Servers. IEEE Trans. Fuzzy Systems, Vol. 7, No. 1, pp. 17-26, 1999.

[24] The Network Simulator - ns-2, http://www.isi.edu/nsnam/ns/.

[25] Fuzzy Logic Tool Box (For use with MATLAB) Users' Guide Version 2, The MathWorks Inc., http://www.mathtools.net/MATLAB/Fuzzy_Logic/. 
Baolin Sun (born in 1963), graduated in Mathematics (The Huazhong Normal University, Wuhan, China) and received Ph.D. in Computer Science (Wuhan University of Technology, Wuhan, China). He is currently a professor, and one of Editorial Board Guest Members of World SciTech R\&D, one of the paper reviewers of Chinese of Journal Electronics, Journal of Electronics \& Information Technology, High Technology Letters, and KGCM, ICCIT program committee members, and also an International Standard Draft organizing members of ISO/IEC JTC1/SC6. His research interests include QoS multicast routing, distributed computing, network optimization and ad hoc networks. Professor Sun has published over 100 research papers and is the author of four books. He was awarded the Province Special Prize by the Hubei Province Government in 2007.

Chao Gui (born in 1964), graduated in Physics (The Wuahn University, Wuhan, China) and received M.S. degree in Electrical Engineering (Wuhan University of Technology, Wuhan, China). $\mathrm{He}$ is currently a associate professor in Computer Science. His research interests include wireless communication, performance analysis and analytical modeling. He has published over 30 research papers.

Qifei Zhang (born in 1977), graduated in Computer Science (The Jianghan University, Wuhan, China) and received Ph.D. in Information and Communication Engineering (Huazhong University of Science and Technology, Wuhan, China). He is currently a lecturer in Computer Science. His research interests include wireless local area networks, Mesh Networks, Network Protocol and Congestion Control. He has published over 30 research papers.

Hua Chen (born in 1977), graduated in graduated in Mathematics (The Huazhong Normal University, Wuhan, China) and received M.S. degree in Mathematics (Huazhong University of Science and Technology, Wuhan, China). She is currently a lecturer in Mathematics. Her research interests include wireless communication, performance analysis, network model and algorithm. She has published over 20 research papers. 